\title{
CUALIFICACIÓN, SOCIALIZACIÓN Y TERCIARIZACIÓN ${ }^{*}$
}

\author{
ENRIC SANCHIS
}

Universidad de Valencia

\section{PALABRAS CLAVE ADICIONALES}

Cualificación técnica, social, explicita, tácita, nominal y efectiva, Género.

\section{ADDITIONAL KEYWORDS}

Technical, social, explicit, tacit, nominal, and Effective skill, Gender.

RESUMEN. Desde hace más de dos décadas, el problema de la cualificación ha pasado a ocupar un lugar central en los debates sobre politicas activas de empleo y estrategias de modernización económica. En estos debates suele darse por supuesto un concepto de cualificación que tiende a ocultar su complejidad. En este artículo se discuten algunos de los problemas que se esconden tras este concepto. Primero se distingue entre la dimensión socio-cultural de la cualificación y la técnico-profesional (que puede descomponerse en cualificación explícita y tácita). Luego se define la cualificación del trabajador (c. efectiva) en relación a la del empleo que ocupa (c. nominal) y se plantea el problema de la confrontación entre ambas. Este problema se aborda considerando la cualificación como resultado de un proceso de construcción social y distinguiendo entre definición técnica y social de la cualificación. Finalmente se analiza la cuestión de cómo y dónde se adquiere la cualificación y se interviene en el debate sobre las relaciones entre ésta y el cambio tecnológico.

SUMMARY. During the last two decades the problem of skill has come to be a key theme in debates about active employment policies and strategies for economic modernization. In such debates the concept of skill tends to be employed in a manner which does not reflect its complexity. The article seeks to remedy this. First it makes the distinction between the socio-cultural and the technical/professional dimensions of the concept ( the technical/professional can be further broken down into explicit and tacit skills.). Secondly, the distinction is made between the skills of the worker (effective) and the skills associated with the job he/she occupies (nominal) and the possibility of a lack of fit between the two. Skills are seen as the product of a process of social construction which is different from its technical definition. Finally the article considers the issue of how and where skills are acquired and examines the discussion on the relationship between skills and technological change.

E-mail: enric.sanchis@uv.es

"Agradezco las observaciones de Miguel Ángel Garcia Calavia y Pere J. Beneyto a una primera versión de este trabajo, que se ha beneficiado igualmente del debate que siguió a su exposición durante un seminario celebrado en la Universidad Autónoma de Barcelona (marzo-2001). La versión definitiva (diciembre de 2001) también es deudora de las sugerencias de un evaluador anónimo.

Revista Internacional de Sociología (RIS)

Tercera Época, $n^{\circ}$ 30, Septiembre-Diciembre, 2001, pp. 139-167. 
R I S

REUISTA INTERNACIONAL DE SOCIOLOCLA

N 30, Septiembre-Diciembre, 2001

ERIC SANCHIS

Desde los inicios de la revolución industrial, los estudiosos de la economía y la sociedad han mostrado un interés recurrente por la importancia de la cualificación de la fuerza de trabajo como factor de desarrollo económico, así como por la relación entre ella y el progreso técnico. La reflexión al respecto puede considerarse ya planteada en las obras de los escoceses A. Smith y A. Ferguson, pasa por la obra de K. Marx y llega hasta nuestros días con la teoría del capital humano y las investigaciones sobre la descualificación de $\mathrm{H}$. Braverman que, como refleja M. A. García Calavia (1999), han dado lugar a una riquísima controversia que sigue abierta en la actualidad.

Desde mediados de los años setenta, con la eclosión del paro masivo y la aplicación de las tecnologías de la información a los procesos productivos, el problema de la cualificación laboral ha pasado a ocupar un lugar central tanto en el debate sobre las políticas activas de empleo como en las estrategias de modernización del tejido productivo. La perentoriedad con que se plantean estas cuestiones en el ámbito de los gobiernos y administraciones públicas, agentes económicos e interlocutores sociales, muchas veces da por descontado el concepto de cualificación, lo que tiende a ocultar su complejidad y multidimensionalidad. Como ha señalado la directora del Instituto Nacional de Cualificaciones, "no es tan fácil, cuando hablamos de cualificación, decir de qué hablamos" (Arbizu, 2000: 30).

El objetivo principal de esta reflexión es, pues, contribuir a una definición sistemática de este concepto, prestando atención particular a la vinculación entre cualificación laboral y proceso de socialización, a la necesidad de entender la cualificación no sólo en términos técnicos sino también sociales y a las transformaciones que experimenta cuando se aplica al trabajo en los servicios. Pero también se ocupará del proceso de adquisición de la cualificación y de las relaciones entre ésta y el cambio tecnológico. La necesidad de que, desde la Sociología del Trabajo, se discuta explícitamente el concepto de cualificación es cada vez más inexcusable. Primero, porque este concepto ha nacido y ha sido aplicado preferentemente en el ámbito del trabajo obrero industrial, actualmente en regresión frente a la expansión del empleo en los servicios. Segundo, porque la relación cada vez más estrecha entre titulaciones educativas y cualificaciones laborales ha dado lugar a problemas tradicionalmente poco estudiados por la Sociología del Trabajo, como es el caso de los conocimientos y destrezas que sólo pueden ser adquiridos al margen del proceso de trabajo. Tercero, porque la aparición y aplicación de dispositivos de erogación de cualificaciones específicamente diseñados para hacer frente al paro estructural ha puesto de manifiesto que la cualificación es algo más que el dominio de ciertos conocimientos y habilidades. 


\section{APROXIMACIÓN AL CONCEPTO DE CUALIFICACIÓN}

\section{Formación y experiencia}

La cualificación puede definirse intuitivamente en función de las dificultades que entraña la ejecución efíciente de una tarea. Así, trabajo cualificado será el que resulta dificil hacer, y no cualificado el que cualquiera puede llevar a cabo. Una definición aparentemente sencilla pero que no puede ocultar, a poco que se reflexione, la complejidad de las cuestiones implicadas.

Casi todos los trabajos requieren una combinación de esfuerzo físico, habilidades manipulatorias y conocimientos. En principio puede convenirse que las tareas que exigen sobre todo esfuerzo físico son las poco o nada cualificadas, mientras que las más cualificadas son las que exigen sobre todo conocimientos. Las habilidades manipulatorias se adquieren con la práctica, es decir con la experiencia laboral, aunque en función de la complejidad de la tarea y de otros factores puede ser necesaria una fase de entrenamiento previo (por razones obvias, ni el cirujano ni el artificiero deben comenzar a adquirir sus especiales habilidades en situaciones de trabajo real). Por lo que se refiere a los conocimientos, puede distinguirse entre los de tipo teórico, técnico o abstracto (incluyendo una manera determinada de razonar o de enfrentarse a los problemas), que requieren un periodo previo de formación, y los de tipo empírico, que dependen igualmente de la experiencia laboral. Como ha señalado A. Touraine (1963), la importancia relativa de los conocimientos teóricos y de la experiencia en la definición de la cualificación depende del tipo de trabajo y de la técnica utilizada para realizarlo.

De acuerdo con estas consideraciones, puede definirse la cualificación - definición ampliamente aceptada que utiliza, entre otros, M. Vernières (1978:22) - como "el producto combinado de la experiencia y la formación", siendo más cualificados los trabajos que requieren procesos más largos de ambas. Por el contrario, trabajo no cualificado es el que se aplica a la ejecución de tareas u operaciones muy simples, con fuerte predominio de actividad física, que cualquiera puede aprender fácilmente; dicho en otros términos, el que consiste en un número restringido de actividades manuales que podrían ser ejecutadas por la mayoría de la gente tras un período breve de adiestramiento. El trabajo parcelado (ciclos cortos y repetitivos de movimientos sencillos) al que ha dado lugar históricamente la racionalización taylorista del proceso de producción, es el ejemplo clásico de trabajo no cualificado.

Todo esto implica que la cualificación que posee un trabajador en un momento dado es función de conocimientos y habilidades adquiridos al margen de la actividad laboral y de la misma experiencia laboral. En consecuencia, el desarrollo profesional de un trabajador puede depender sobre todo de la experiencia, sobre todo de conocimientos que no se adquieren en el trabajo, o 
bien de cierta combinación de ambos elementos. De donce se deduce que hay trabajos cualificantes, que permiten progresar en la carrera laboral conduciendo al desempeño de trabajos más cualificados, y trabajos que no enseñan nada y que pueden acabar descualificando a quien lleva mucho tiempo haciéndolos. Muchas veces el problema del mercado de trabajo secundario es más esto que los bajos salarios o la inestabilidad del empleo. Es el caso de muchos jóvenes cuyo primer empleo no es el escalón inicial de una carrera laboral sino la entrada a una vía sin salida. Como ha señalado I. García Espejo (1999:214-15), la inserción de los jóvenes españoles en el mercado de trabajo se caracteriza por la elevada rotación laboral, favorecida por las políticas de empleo puestas en marcha durante los últimos años, así como por el acceso a puestos "de bajo nivel de cualificación, para cuyo desempeño no se requiere prácticamente de un aprendizaje", mientras que "la adquisición de cualificaciones en el trabajo es el factor que ejerce efectos más beneficiosos en el inicio y la consolidación de una carrera profesional, por encima de la mera acumulación de experiencia laboral o de credenciales educativas". Así, frente al itinerario clásico de inserción socio-laboral que tiene su punto de partida en el sistema educativo y su punto de llegada en el empleo estable y la independencia familiar, tras un período relativamente breve de precariedad laboral, se ha abierto otro en el que la acumulación de las experiencias laborales erráticas propias del mercado de trabajo secundario no permite acumular un saber profesional a partir del cual se pueda construir un curriculum y alcanzar la emancipación característica de la vida adulta. En consecuencia, la España finisecular ha alumbrado un hecho social casi único en el contexto de las sociedades avanzadas: un nuevo tipo de adultos jóvenes cuya identidad social se define cada vez menos en relación al trabajo y cada vez más en relación a un estilo de vida basado en ciertos consumos compulsivos y en la prolongación de la estancia en el domicilio paterno. Según el Informe Juventud en España 2000, el momento de tránsito a la edad adulta tiende a posponerse y sólo el $29 \%$ de los jóvenes (población de 15 a 29 años) son económicamente autónomos; a los 25 años sólo el $32 \%$ están emancipados económicamente, y a los 29 aún no lo está el $28 \%$.

\section{Cualificación técnico-profesional y socio-cultural}

Resistencia fisica, habilidades manipulatorias, cierto tipo de conocimientos, son factores que forman parte de lo que podríamos llamar la dimensión técnicoprofesional de la cualificación. Pero la actividad laboral suele ejecutarse dentro de una unidad productiva que es a la vez un sistema técnico y un sistema social; para desempeñarla con eficacia es necesario también adaptarse a una organización, aceptar unas normas de funcionamiento, compartir unos valores, mantener ciertas actitudes frente al trabajo, ser capaz de emitir mensajes inteligibles y de comprender los que se dirigen a uno; en una palabra: hay que 
estar socializado para el trabajo. Todos estos factores pueden ser conceptualizados como la dimensión socio-cultural de la cualificación. Si la dimensión técnicoprofesional remite sobre todo a la competencia para llevar a cabo una tarea, la dimensión socio-cultural tiene que ver también con la disponibilidad a hacerlo bajo las condiciones salariales y organizativas exigidas por el empleador. La primera dimensión constituye lo que Offe (1977:169-170) denomina el aspecto "material" de la cualificación, mientras que la segunda constituye lo que denomina el aspecto "social".

No debe confundirse la dimensión socio-cultural de la cualificación con las habilidades sociales que requieren muchos de los empleos localizados en el sector servicios para ser desempeñados con eficacia. Mientras los trabajos desarrollados en el ámbito de la producción de bienes suelen exigir la manipulación de objetos, los trabajos de prestación de servicios requieren (además o exclusivamente) contacto con personas, ya sean clientes, usuarios 0 pacientes. En tal caso la cualificación incluye el dominio de una serie de destrezas relacionales que forman parte de su dimensión técnico-profesional.

La dimensión técnico-profesional de la cualificación puede ser concebida como un agregado de elementos reconducibles a dos grandes tipos. Inspirándome en Coller (1997:108-109) y en los autores por él citados, llamaré cualificación explícita al primer tipo, y cualificación tácita al segundo. La cualificación explícita está constituida por aquellos elementos medibles (objetivables) de la misma: titulaciones educativas y formativas, conocimiento de operaciones concretas, etc. Este tipo de cualificación suele utilizarse para ordenar jerárquicamente (y salarialmente) los puestos de trabajo. La cualificación tácita está constituida por aquellos conocimientos y habilidades derivados de la experiencia laboral y que por tanto no se prestan fácilmente a la objetivación. Esta cualificación se basa en la familiaridad con el proceso de trabajo y con los materiales utilizados durante el mismo e incluye "cierta habilidad para identificar fallos en el funcionamiento de las líneas de producción".

Resumamos lo dicho y simplifiquemos denominaciones para ganar claridad. Hay una cualificación técnica y otra cultural. La cualificación técnica es en parte explícita (objetivable) y en parte tácita (no objetivable: conocimientos operativos y habilidades manipulatorias adquiridos durante el proceso de trabajo). Muchos empleos requieren además habilidades sociales que forman parte de la cualificación técnica.

Pero este esquema simple que pretende ser claro no resuelve algunas cuestiones que requieren un análisis más fino. En primer lugar, la distinción tácito/explícito podría aplicarse también a la cualificación cultural. Puede presumirse que ésta es casi por definición sobre todo tácita y que está relacionada no sólo con el proceso de socialización laboral sino también con el proceso de socialización tout court. Por tanto la cualificación tácita no consiste sólo en conocimientos operativos y habilidades manipulatorias y no puede adquirirse 
sólo en el puesto de trabajo. Por otra parte, las habilidades sociales forman parte de la cualificación técnica, pero su naturaleza es muy diferente. Probablemente son más tácitas que explícitas, pero posiblemente tienen más que ver con el proceso de socialización general (familia, escuela, grupos de iguales...) que con la experiencia laboral. Estas cuestiones tienden a obviarse al hablar de cualificación; probablemente porque se dan por descontadas considerando que forman parte de ciertas culturas del trabajo y que su transmisión intra e intergeneracional está garantizada. Sin embargo Braverman (1974: 96) ya advirtió que la habituación de los trabajadores al modo de producción capitalista debe ser renovada con cada generación, y en las circunstancias actuales puede sospecharse que la transmisión intergeneracional de la cualificación cultural y de las habilidades sociales es todavía más problemática: los hijos de la clase obrera trabajan o quieren trabajar cada vez más en el sector servicios, donde las habilidades sociales son más importantes que en la industria; pero estas habilidades son típicas de las clases medias y no puede darse por descontado que los hijos de la clase obrera las tengan. Es por esto por lo que el problema de la cualificación no puede seguir planteándose sin considerar el problema de la socialización y el significado del proceso de terciarización.

\section{Cualificación efectiva y nominal}

Hasta ahora se ha estado utilizando la expresión cualificación laboral en un sentido bivalente para poder relacionarla, por una parte, con las competencias poseídas por el trabajador, y por otra con las exigencias del puesto de trabajo que ocupa. En realidad se trata de dos cosas diferentes aunque fuertemente relacionadas. Como señala Touraine, parece difícil buscar fuera del mismo individuo el principio de definición de su cualificación, pero hay que renunciar a definirla al margen de la situación en que se ejerce. En todo caso, el hecho es que quien vende su fuerza de trabajo define la cualificación de manera diferente a quien la compra.

Para el trabajador, la cualificación laboral es el conjunto de conocimientos, habilidades y experiencias adquiridos antes y durante su itinerario laboral; los que aplica en su empleo actual, los que aplicó en otros empleos e incluso los que no ha llegado a aplicar nunca pero podría poner en práctica si las circunstancias lo exigiesen, y llega a incluir hasta sus características físicas y de personalidad. A la cualificación laboral entendida desde la perspectiva del trabajador se la suele denominar cualificación efectiva. Por el contrario, el empleador define la cualificación a partir de las características del empleo que necesita cubrir. Estas características se concretan después de haber tomado una opción técnica y organizativa, en función de la cual el trabajo necesario para llevar a cabo un proceso productivo dado aparece desagregado en una sucesión de empleos que se definen por la función a desempeñar y a los que se les atribuye una 
cualificación determinada. A la cualificación entendida en este sentido se la suele denominar cualificación nominal, que es la única que en principio estará dispuesto a reconocer formalmente el empresario.

En realidad el empleador difícilmente tomará las decisiones técnicas y organizativas que determinan las características de los diferentes empleos sin tener en cuenta la cantidad y tipo de fuerza de trabajo disponible en el mercado, pero habitualmente existirá una diferencia entre la cualificación que un empresario atribuya en un primer momento a un puesto dado y la considerada necesaria para desempeñarlo con eficacia por parte del trabajador que lo ocupe. Dicho en otras palabras, el reconocimiento formal de la cualificación efectiva siempre será objeto de regateo entre las partes, ya que de él depende también la retribución del trabajador. Factores como el peso que tienen las organizaciones sindicales y los grupos profesionales, la empresa, el sistema educativo y otros dispositivos de formación en la definición de las cualificaciones, así como las tasas de desempleo de los diferentes colectivos que integran la fuerza de trabajo, tenderán a reducir o alargar la distancia entre la cualificación efectiva y la nominal. El carácter subjetivo de la cualificación tácita (que lógicamente forma parte de la cualificación efectiva) también dificultará la equiparación entre ésta y la cualificación nominal. De hecho, la cualificación tácita sólo será reconocida formalmente si los trabajadores pueden imponerla como criterio de clasificación profesional (por ejemplo asignando un valor a la antigüedad en el puesto). En general puede afirmarse que la distancia será menor cuanto más desarrollada esté la democracia económica.

Si se acepta la posibilidad de definir de manera objetiva e independiente la cualificación del trabajador y la del puesto que ocupa, la comparación entre ambas determina tres situaciones posibles: 1) Equilibrio. 2) Sobrecualificación: el trabajador está más cualificado que el empleo. Es el caso de muchos licenciados que ocupan puestos que no requieren su nivel de titulación; situación que no debe confundirse con la de aquellos universitarios que, a causa del paro estructural, se ven obligados a aceptar empleos de cualificación superior para cuyo desempeño no se exige formalmente la licenciatura. 3) Infracualificación; es el caso contrario, que o bien es teóricamente imposible o bien da lugar a un desempeño ineficiente del puesto de trabajo.

\section{SOCIALIZACIÓN Y CUALIFICACIÓN}

Afirmar que la cualificación laboral de un individuo se fundamenta en su socialización previa puede parecer una tautología. Sin embargo, en el contexto de paro masivo y transición de una economía industrial a otra terciaria, algunos de los interrogantes planteados en torno al problema de la cualificación tienen más que ver con el proceso de socialización que con otra cosa. Quien no esté 
RIS

REVISTA INTERNACIONAL DE SOCIOLOGIA

No 30, Septiembre-Diciembre. 2001

ERIC SANCHIS

familiarizado con lo que podría denominarse el ambiente social típico de las clases medias y no haya interiorizado ciertas habilidades relacionales durante su infancia-adolescencia, tendrá más dificultades para desempeñar su rol laboral en actividades como la docencia, la sanidad o el comercio al por menor. Y, como se verá un poco más adelante, en algunos casos determinadas capacidades estrictamente técnicas también quedan bajo la influencia directa del proceso de socialización. Pero es en relación con la cualificación cultural donde la importancia de la socialización parece más evidente. Pueden distinguirse tres ámbitos problemáticos.

En primer lugar, las dificultades de control de la fuerza de trabajo cuando está constituida por trabajadores adultos que se incorporan por primera vez al empleo urbano industrial tras haber sido socializados en un entorno agrícola rural. En este sentido es obligado recordar las observaciones de E. P. Thomson (1984:271 y ss.) sobre la necesidad, en los albores de la revolución industrial, de construir e inculcar una disciplina del tiempo y del trabajo fabril que tuvo que enfrentarse a la cultura popular y a los antiguos hábitos laborales; tanto una como los otros sólo después de varias décadas comenzaron a ser sensibles a unos estímulos salariales que, según la hipótesis del comportamiento económico racional, deberían haber tenido efectos inmediatos sobre las primeras generaciones de trabajadores industriales. Y la pregunta que surge es: ¿no puede establecerse un paralelismo entre todo esto y las dificultades de reabsorción del excedente obrero industrial en los nuevos empleos terciarios, que tan bien ha sabido captar el cineasta Peter Cattaneo en Full Monty?

En segundo lugar, la integración de las nuevas generaciones en la vida activa. Este tema ha sido tratado en profundidad en E. Sanchis (1991:177-229). Pueden distinguirse dos casos típicos. El primero es el de los niños de clases populares en sociedad tradicional o de capitalismo temprano, donde el problema tiende a reducirse al de la adaptación o ajuste al medio laboral, sin que la adquisición de las competencias técnico-profesionales plantee mayores dificultades. La experiencia laboral y vital del personaje central de un estudio antropológico de J. Frigolé (un pequeño campesino y jornalero murciano, nacido en 1901, que comenzó a trabajar a los ocho años de mozo de un aparcero) ejemplifica perfectamente este caso. Una experiencia compartida por gran parte de la población rural española hasta bien entrada la segunda mitad del siglo XX, que ha sido reflejada por A. Muñoz Molina (1991:145-146) en una de sus novelas:

Porque la infancia había terminado tan prematuramente para ellos que luego casi no recordaban haberla conocido: fueron apartados de la escuela por la llegada de la guerra y un día descubrieron que faltaba el padre en la casa y que para sobrevivir tenían que abandonar los juegos en la calle igual que unos meses atrás habían abandonado las aulas y aprender la disciplina de un trabajo que les rompía los huesos y les desollaba las manos [...] Crecieron en la incertidumbre de la 
guerra y en la penuria del racionamiento y se aclimataron a ellas como si fueran los atributos naturales de la vida, [...] y sólo muchos años después, cuando han notado que envejecen antes de tiempo, descubren que no en su memoria, sino en el dolor de las rodillas y en la desconcertante fragilidad de sus vértebras, ha perdurado la injuria de una temprana expulsión de la que ni siquiera se quejaron cuando la sufrían, aletargados en el fatalismo y en la irrealidad de la infancia, como cuando los despertaban antes del amanecer para que fueran al campo y bajaban medio dormidos por los caminos de las huertas llevando al hombro una hoz o una azada que apenas sabían manejar.

Como ha señalado $\mathrm{Ph}$. Ariès (1973), en la sociedad tradicional la infancia se reduce al mínimo y el niño es mezclado enseguida con los adultos, con quienes comparte su trabajo y juegos. A los siete años más o menos el niño se convierte en un hombre joven sin pasar por la etapa de la juventud. Su escuela será la coexistencia con los adultos, de quienes aprende las cosas que tiene que saber ayudándoles a hacerlas. En el mundo rural, siete años es una edad excelente para empezar a cuidar los corderos, con nueve años ya puede uno encargarse de los cerdos y a los doce comenzar a arar con los bueyes; al llegar a los dieciséis se participará en la limpieza de la granja, pero aún no se habrá aprendido a sembrar (Crubellier, 1979:118). Sí se habrá aprendido, muchos años antes, que al trabajo se le da un valor absoluto en el mundo adulto, donde la pereza es casi el único verdadero pecado capital (Handman, 1983:137). Pero el choque con el trabajo industrial no es menor. En la monografia elaborada por M. Mietto y M. G. Ruggerini (1988:187-190) sobre las experiencias laborales, vitales y sindicales de los obreros de una fábrica italiana durante los años cincuenta del siglo XX, los escasos párrafos dedicados a evocar el primer encuentro con la fábrica son sin embargo suficientes para comprobar que se trata de una experiencia traumática de la que se desea escapar, que se sufre una fuerte sensación de angustia y que el mundo que se acaba de descubrir es percibido como si se tratara de una cárcel. Ahora bien, lo que interesa destacar de todo esto es que la integración en la vida activa, tanto en medio rural como industrial, es más un proceso de aprendizaje de pautas culturales que no de conocimientos técnicos o habilidades manipulatorias.

El segundo caso típico es el de los jóvenes de sociedad moderna y de capitalismo maduro procedentes de estratos superiores de clase obrera e inferiores de clases medias que se incorporan al trabajo manual tras un proceso de escolarización más o menos largo. Aquí el problema es que el medio cultural familiar, consumista y escolar en el que han sido socializados resulta parcialmente extraño a la cultura específica del trabajo industrial. Aparece una contradicción entre los valores, orientación al trabajo y expectativas de movilidad ocupacional y social, por un lado, y la realidad del mundo del trabajo manual por otro. Las empresas que se quejan de la falta de competencia de los jóvenes y que utilizan 
como primer criterio de selección de personal la formación en virtudes humanas para el trabajo, están reconociendo tácitamente que su problema no es que no encuentren trabajadores lo bastante eficientes a causa de sus carencias técnico-profesionales, sino que no encuentran trabajadores lo bastante obedientes para integrarse en el proceso de trabajo sin cuestionar la organización jerárquica y disciplinaria de la unidad productiva. Dicho en palabras de un empresario del sector electrónico: antes que una cualificación se busca más bien una persona voluntariosa que esté dispuesta a trabajar (J.J. Castillo et al., 2000:39). Probablemente estas consideraciones pueden hacerse extensivas a los empleos menos atractivos del sector servicios. La diferencia esencial entre los dos casos típicos señalados radica en que el primero se desarrolla en un contexto de hambre y penuria generalizada, que como se sabe es un mecanismo de socialización muy efectivo.

El tercer ámbito problemático se refiere a la cultura obrera, entendida ésta específicamente como conjunto de valores y actitudes ante el trabajo alternativos a la cultura de la empresa. Del mismo trabajo de Mietto y Ruggerini puede deducirse el dilema permanente a que se enfrenta el trabajador manual. Por una parte siente el impulso de exhibir su profesionalidad, de reafirmar el orgullo del oficio cruzando la frontera (impuesta por la organización científica del trabajo) entre concepción y ejecución, de colaborar lealmente en el proceso productivo contribuyendo al progreso tecnológico y al desarrollo industrial, demostrando así un sentido de la responsabilidad que, en el caso del militante político y sindical, llega incluso a considerarse como un deber, porque tiene que dar ejemplo a sus compañeros. Por otra, teme que esta disponibilidad sin reservas acabe reduciendo su capacidad de control sobre el proceso de trabajo y debilitando su posición en el conflicto industrial.

\section{LA CONSTRUCCIÓN SOCIAL DE LA CUALIFICACIÓN}

¿Por qué los empleos masculinos son sistemáticamente más cualificados y están sistemáticamente mejor pagados que los femeninos? Sustitúyase el binomio masculino-femenino por blanco-negro, adulto-joven, autóctono-inmigrado, etc. y reformúlese la pregunta. ¿En qué medida la cualificación formalmente reconocida integra la cualificación tácita?, ¿hasta qué punto las diferencias en cualificación explican las diferencias salariales? Un economista ortodoxo intentaría responder a estas preguntas en clave de capital humano y de productividad del trabajo. Es decir, de acuerdo con el supuesto básico de lo que C. A. Castillo Mendoza y E. Terrén Lalana (1994:78) han llamado el paradigma clásico de la teoría de la cualificación: la cualificación es entendida básicamente como un capital característico del factor trabajo cuyo precio puede medirse objetivamente por su productividad marginal relativa y traducirse directamente 
en el salario. Se parte, por tanto, de una absoluta transparencia de la relación formación-cualificación-salario, en virtud de la cual las "cualidades" individuales se transforman de manera armónica y directa en jerarquías salariales $y$, consiguientemente, sociales. Pero a cualquier observador un poco más sensible a la realidad de los hechos sociales esta respuesta le dejaría más bien insatisfecho.

$\mathrm{Al}$ hablar de cualificación hay que distinguir claramente entre lo que son competencias definidas objetivamente y el nivel profesional socialmente definido, que puede ser más o menos independiente de las competencias objetivas y que es en última instancia el que determina el nivel de remuneración. Una cosa es la definición "técnica" de la cualificación (nivel educativo, habilidades, experiencia, etc.), y otra distinta la definición "social", que es la "resultante de las negociaciones entre los distintos colectivos implicados en la producción y el mantenimiento del prestigio de la ocupación. Negociaciones que se entablan para llegar a un acuerdo sobre la denominación, definición y certificación y para determinar las entidades de transmisión y las condiciones de valoración" (Gómez Bueno, 2000:36). Como afirma S. Wood (1994:302), "para un sociólogo todos los empleos y cualificaciones están construidos socialmente en el sentido de que no están determinados por ninguna tecnología llovida del cielo. La cuestión es hasta qué punto la etiqueta de cualificados que se pone a ciertos empleos es independiente de la tecnología". La consideración de que el nivel profesional socialmente definido de un empleo es el resultado de un proceso de construcción social, permite buscar respuestas más solventes a aquellas preguntas.

Dando por descontada la cualificación cultural y prescindiendo de factores como penosidad, peligrosidad, responsabilidad, nocturnidad, etc., en principio podemos decir que el precio del trabajo cualificado (como el de cualquier otra mercancía) es función de su coste de producción (tiempo necesario para proveerse de la experiencia y formación correspondiente) y de su escasez relativa (cotización en el mercado). Así, el dominio de la lectura, escritura y las cuatro reglas de cálculo será una cualidad preciosa o banal según el nivel de alfabetización de la población. Sin embargo, una explicación más realista debe tener en cuenta también las diferentes estrategias de "internalización" de ciertos mercados laborales puestas en marcha ya sea por la empresa (para quebrar la cohesión y reducir el poder de los trabajadores), ya sea por ciertos grupos de trabajadores para preservar sus condiciones de trabajo de la competencia exacerbada. En este último caso, la acción puede orientarse directamente contra la empresa o bien contra otros grupos de trabajadores. Las dificultades crecientes de acceso a ciertas profesiones para cuyo ejercicio se exigen credenciales educativas cada vez más altas que no guardan proporción con la complejidad técnica correspondiente, fenómeno explicado por R. Collins (1989) en un libro que puede considerarse ya como un clásico de la sociología de la 
RIS

REVISTA INTERNACIONAL DE SOCIOLOGLA

№30. Septiembre-Diciembre, 2001

estratificación, ilustran perfectamente lo que se pretende argumentar. Serían las estrategias de "blindaje" de determinadas ocupaciones las que explicarían que las cualificaciones consideradas típicamente masculinas fueran definidas socialmente como superiores a las consideradas típicamente femeninas. Esto es relativamente sencillo cuando se trata de cualificaciones no adquiridas en dispositivos formales, bien porque son tácitas, bien porque han sido adquiridas durante el proceso de socialización tradicional de las niñas en la familia. Este último es el caso de Marina, la joven trabajadora de una fábrica de tejidos de quinientos empleados entrevistada por A. Moncada (1979:126). Fue su madre quien le enseñó a coser, cortar trajes, etc., "un entrenamiento estupendo" para su trabajo, "mucho mejor que los dos cursos de corte y confección" que hizo antes de empezar a trabajar: "Mi madre tiene un instinto para la estética y el aprovechamiento de los retales que es bárbaro. Por lo visto las niñas manchegas [como su madre] se pasaban la vida con la aguja en la mano".

En definitiva, la consideración de la cualificación como resultado de un proceso de construcción social permite albergar dudas sobre cuestiones como la supuesta infracualificación de muchos empleos basura cubiertos por jóvenes en el sector servicios y preguntarse si ciertos empleos son femeninos porque carecen de cualificación o carecen de cualificación porque son femeninos. Como señala Gómez Bueno (p. 35), "el concepto de cualificación es fruto de una construcción social que resulta de una serie de luchas entre los diferentes sectores sociales implicados en la definición del trabajo: luchas simbólicas pero también luchas político-económicas, porque lo que está en juego es la relación de fuerzas entre los diversos grupos - entre empresarios y trabajadores, así como entre los diversos grupos de trabajadores-y sus derechos diferenciales de acceso a recursos materiales".

Los estudios de género están contribuyendo de manera decisiva a desvelar el carácter reduccionista de un concepto de cualificación construido sólo en función de factores técnicos así como la influencia que variables sociológicas y políticas ejercen en el mismo. Gómez Bueno ha examinado la Clasificación Nacional de Ocupaciones española vigente desde 1994 y el Convenio Colectivo para la Industria Textil y de la Confección que se aplicó durante la segunda mitad de los años noventa, a fin de descubrir posibles prácticas de discriminación salarial indirecta de las trabajadoras en los procesos de producción-adjudicación de cualificación a las ocupaciones. En particular fija su atención en la distinción entre ocupaciones cualificadas y no cualificadas.

En primer lugar analiza las novedades registradas en la Clasificación Nacional de Ocupaciones actual respecto a la anterior, que era de 1979. En general se aprecia que los cambios en la denominación de las ocupaciones van en sentido recualificador, lo que atribuye a la presión de los colegios profesionales (en los niveles superiores de la jerarquía ocupacional) o de los sindicatos (en otros niveles). Es muy significativa la desaparición del término obrero, que ha sido 
sustituido por trabajador, operador o peón: "estos cambios de denominación forman parte de una estrategia simbólica de valorización consistente en etiquetarse con un nombre prestigioso". Las denominaciones adjudicadas a algunas nuevas ocupaciones que aparecen en la clasificación de 1994 apuntan en la misma dirección: profesional de..., técnico en...

La Clasificación Nacional de Ocupaciones, al igual que la International Standard Classification of Occupations, utiliza como criterio principal para definir las categorías ocupacionales las titulaciones educativas, si bien a veces también se tienen en cuenta criterios de autoridad. Esto permite distinguir entre ocupaciones cualificadas y no cualificadas, aunque muchas ocupaciones escapan a esta dicotomía. Sin embargo, la Clasificación no refleja con precisión la realidad de la empresa, es decir, cómo se estructuran funcional y jerárquicamente los puestos de trabajo que existen en ella. En cambio, "los convenios colectivos nos revelan las categorías ocupacionales definidas y aprobadas en cada sector productivo, los criterios empleados para la valoración de cada categoría ocupacional, así como los coeficientes y salarios correspondientes a cada una de ellas". Por tanto es en el convenio colectivo, que regula las relaciones entre empleadores y empleados, donde se puede analizar mejor el proceso de construcción social de la cualificación. Si además se escoge el de un sector altamente feminizado, como es el caso del textil-confección, al mismo tiempo podrá estudiarse la influencia de la variable género en ese proceso.

Los criterios que se utilizan en la industria textil y de la confección para la asignación de niveles profesionales dentro de cada categoría ocupacional son autonomía, mando, responsabilidad, iniciativa, complejidad y formación (que tiene en cuenta el nivel educativo y, sobre todo, la experiencia adquirida en el puesto de trabajo). A juicio de Gómez Bueno, estos criterios son tan amplios y ambiguos que dejan un gran margen de maniobra para ser aplicados, y el resultado final es que los empleos ocupados por mujeres son catalogados entre los menos cualificados y peor retribuidos. Los coeficientes asignados a las categorías ocupacionales más feminizadas suelen ser más bajos que los correspondientes a las otras categorías. "Estos coeficientes son los que determinan los salarios y hay algo sospechoso en ellos. Igual que lo hay en las diferencias salariales que se establecen entre subsectores: [...] La confección es el subsector peor remunerado y también el más feminizado". Además, el dato paradójico de que así en el textil como en la confección el nivel educativo medio sea superior entre las mujeres pone de manifiesto que "la división social del trabajo no es neutral respecto al género: la segregación tanto horizontal como vertical refuerza el argumento de que las cualificaciones no son socialmente neutrales". ¿Cómo explicar si no el hecho de que, en la confección, la costura (desempeñada mayoritariamente por mujeres) no esté cualificada y el corte (desempeñado por varones) sí lo esté?

En última instancia, la variable que explica la distribución asimétrica de las 
cualificaciones por género es el papel secundario (o inexistente) que desempeñan las mujeres en la negociación colectiva, que no es sino un reflejo de la posición secundaria que ocupan en la sociedad. "Los criterios de valoración de la cualificación de las ocupaciones no son neutrales. Por el contrario, son un reducto de las antiguas características del trabajo obrero en masculino y, aunque los trabajos han cambiado, los criterios de valoración no lo han hecho". Gómez Bueno concluye su análisis apoyándose en M. Maruani (1991: 133) para explicar que desde que las mujeres comenzaron a incorporarse masivamente al mercado laboral se han ido estableciendo, primero, distancias entre el ámbito masculino y el femenino, y después criterios diferenciados de valoración de las cualificaciones. La cualificación aparece así como una cuestión sexuada: la distinción masculino/femenino es el eje central en torno al cual se construye la noción misma de cualificación. En consecuencia, la definición de la cualificación no es una cuestión técnica: es el resultado de conflictos y negociaciones entre grupos sociales en los que las mujeres suelen salir perdiendo.

\section{LA ADQUISICIÓN DE LA CUALIFICACIÓN}

¿Cómo y dónde se adquiere la cualificación? Mientras que tradicionalmente la inmensa mayoría de las cualificaciones laborales se adquirían en la familia y en la misma unidad productiva, en la actualidad el sistema educativo y otros dispositivos extralaborales de formación desempeñan un papel cada vez más importante en este dominio. Ello se debe a la reducción de las unidades productivas de carácter familiar, a la paulatina desaparición, por diversos motivos, de las escuelas de aprendices que solía haber en muchas grandes empresas (A. Lope y A. Martín Artiles, 1993:76) y a la misma generalización y ampliación del sistema educativo (vinculada paradójicamente, por una parte, a la extensión de los derechos de ciudadanía, y por otra a la eclosión del paro masivo). Además, la rapidez de los cambios tecnológicos y organizativos que experimenta el mundo del trabajo exige períodos formativos más amplios como garantía de una buena capacidad de adaptación a unas circunstancias productivas que van a modificarse más de una vez a lo largo de la vida activa de los trabajadores. Desde el punto de vista del trabajador, tal exigencia es evidente. Si las cualificaciones adquiridas en el puesto de trabajo tienden a quedar obsoletas a causa de los cambios, sus posibilidades de hacer carrera tanto dentro de la empresa como cambiando de empresa dependerán en mayor medida de la solidez de su base educativa. Asimismo, la cada vez más reclamada polivalencia y flexibilidad funcional de los trabajadores requiere igualmente una buena base educativa. En todo caso, en las sociedades modernas la interposición entre la familia y la unidad productiva de una tercera instancia de erogación de cualificaciones plantea la cuestión de las relaciones entre ellas. 
Por lo que se refiere a la familia, se supone y en parte es cierto que pierde peso en favor del sistema educativo. Ahora bien, si recordamos que la cualificación laboral incluye una dimensión socio-cultural, que implica socialización para el trabajo y que requiere cada vez más habilidades sociales, tendremos que reconocer que la familia continúa desempeñando un papel fundamental. Existen correlaciones evidentes entre familia de origen, trayectoria escolar e itinerario laboral; algo que los estudios sobre estratificación social y la sociología de la educación han tenido siempre muy claro, y que la sociología del trabajo ha tendido a obviar. Al hablar de cualificaciones laborales hay que dar más importancia a esta cuestión.

Las relaciones entre sistema educativo y sistema productivo son problemáticas por naturaleza. Tradicionalmente agencia de adoctrinamiento por antonomasia en clave religiosa o en clave política (formar buenos cristianos o formar buenos ciudadanos), con la consolidación de la democracia política y de la economía capitalista, el sistema educativo se ha visto sometido a dos exigencias en parte contradictorias: desde la sociedad se le reclama que haga efectivo el principio de igualdad de oportunidades; desde la economía, que forme buenos trabajadores. Teniendo en cuenta que parte de la cualificación depende de la propia experiencia laboral, la pretensión de algunas empresas (en particular las de tamaño reducido) de que se les sirvan trabajadores a la carta hay que valorarla como poco realista. Quiero pensar que, en la sociedad hacia la que vamos, sin ciudadanos más y mejor educados cada vez será más difícil obtener trabajadores bien cualificados; y creo que este tipo de ciudadanos incluso puede aumentar la eficiencia del sistema productivo en su conjunto, al menos de un sistema productivo en el que la democracia económica ocupe un lugar más relevante. Pero la controversia sobre el papel a desempeñar por el sistema educativo sigue sin estar cerrada; estudiosos como W. Streeck (1992), señalan la necesidad de que las empresas, precisamente a causa de la rapidez del cambio y de la imposibilidad de que el sistema educativo siga su ritmo, asuman un papel más importante en la cualificación de sus trabajadores.

En todo caso, la cualificación en el empleo sigue siendo fundamental, tanto por lo que se refiere a la adquisición de conocimientos empíricos y habilidades específicas como, sobre todo, en relación con la transmisión de la cualificación tácita por parte del trabajador experimentado, quien debe hacer frente a una delicada disyuntiva. Por una parte, sólo estará dispuesto a enseñar los secretos del oficio a su inexperto compañero si tiene la seguridad de que no va a ser sustituido por éste en cuanto acabe su aprendizaje (por ejemplo porque es más joven y barato). Por otra, si pretende que su cualificación tácita sea reconocida formalmente, debe estar dispuesto a objetivarla, en la medida de lo posible, para que pueda ser socializada, es decir integrada en el know-how de la empresa, y por tanto debe estar dispuesto a ceder parte de su poder sobre el proceso de trabajo. El caso analizado por X. Coller (pp. 70-71) al estudiar los efectos de la innovación 
RIS

RETISTA INTERNACIONAL DE SOCIOLOGIA

$N^{*}$ 30, Septiembre-Diciembre. 2001

tecnológica sobre el trabajo ilustra claramente todo esto.

Se trata de una trabajadora de la sección de taxografia de una empresa, encargada de dar las órdenes a la computadora acerca del tipo de tela, número de serie del patrón del corte, cantidad de piezas que tenía que dibujar y luego cortar, etc. Antes "la trabajadora realizaba estas tareas manualmente y a lo largo de su vida profesional había desarrollado una habilidad especial para encajar las piezas de diferentes modelos de manera que se desperdiciara la menor cantidad posible de tela. Esta cualificación tácita se basaba en el conocimiento de cada uno de los modelos (y hay más de cuatrocientos) y de las piezas que lo componen". La introducción de los microprocesadores - el programa informático se diseñó recurriendo a su experiencia - sacó a la luz esta cualificación tácita, la convirtió en un bien disponible para cualquier persona y (afirma Coller) privó a la trabajadora de cualquier posibilidad de resistencia. Según explica la tâxógrafa en una de las entrevistas, desde que habían informatizado el trabajo ganaba más dinero y estaba mejor considerada en la empresa, pero el trabajo era más estresante: la objetivación de la cualificación tácita le impedía arreglarse la producción a su manera, ya que era el ordenador el que dictaba el ritmo.

Comentario aparte merece la formación profesional, concepto genérico que en España agrupa tres dispositivos de erogación de cualificaciones laborales: la Formación Profesional Reglada (FPR), la Formación Profesional Ocupacional (FPO) y la Formación Profesional Continua (FPC), siendo ésta la que ha experimentado una mayor expansión durante los últimos años. Así, mientras antes de 1993 pasaban por ella menos de 100.000 trabajadores al año, en 1996 participaron en este tipo de programas formativos 1.300 .000 trabajadores (P.J. Beneyto y P. Guillén, 1998:23). En teoría, estos dispositivos se diferencian por factores como la clientela preferente que pretenden atender, la financiación o la adscripción administrativa; en la práctica no es raro que algunas de estas diferencias tiendan a diluirse. La FPR está integrada dentro del sistema educativo y diseñada para jóvenes en la edad correspondiente, pero tanto en este dispositivo como en otras parcelas del sistema educativo ha dejado de ser anecdótica la presencia de adultos (ocupados, parados o inactivos). La FPO depende de la Administración laboral y su cliente típico ideal es el parado que ha perdido un empleo, pero una cuota relevante de sus alumnos son jóvenes en busca de primer empleo y con trayectorias educativas deficientes. La FPC, finalmente, se dirige a los ocupados que necesitan ampliar o modificar sus cualificaciones. Siendo así, parece lógico que sea asumida por las mismas empresas y los trabajadores directamente implicados (o sus organizaciones sindicales). Sin embargo en España, y también en otros países, la Administración laboral desempeña un papel muy importante en este dominio.

Con estructura y resultados desiguales según los países, a lo largo de las últimas décadas en Europa se han venido configurando diferentes modelos de FPC: "creados ex novo en algunos casos y resultado de reformas sucesivas en 
otros; con regulación estatal y orientados fundamentalmente a las necesidades de las empresas (Francia), regidos por el mercado y gestionados por entidades privadas (Inglaterra), gestionados paritariamente por empresarios y trabajadores (Dinamarca) o de carácter mixto (Alemania); centralizados o diferenciados territorialmente (Italia), etc." Por lo que se refiere a España, la tardía configuración de su sistema de FPC ha permitido "construir un modelo que integrase de forma dinámica los elementos más positivos de la experiencia europea [...que...] intenta satisfacer las necesidades y requerimientos tanto de los trabajadores como de sus empresas y del conjunto del aparato productivo, y lo hace incorporando como valor añadido su diseño negociado y gestión paritaria" (ibidem: 26, 28).

En el debate sobre las funciones que debe desempeñar el sistema educativo en relación con las cualificaciones laborales, una de las cuestiones más polémicas es la de hasta dónde debe llegar la educación, dónde comienza la responsabilidad de otros dispositivos formativos y a partir de qué punto es la misma empresa (o el trabajador) quien debe organizar su propio dispositivo. Esta cuestión, que planteada en términos menos abstractos debe tener en cuenta variables como el tamaño de la empresa, remite a otra mucho más prosaica: la de quién debe asumir el coste de la cualificación, y deviene particularmente polémica en el caso de la FPC, lo cual no es sino un reflejo de la diversidad de intereses y puntos de vista con que empresarios, sindicatos y administraciones públicas -más allá de las declaraciones retóricas en cuanto a importancia, necesidad y ventajas - contemplan los objetivos y los medios de la FPC. "Para los primeros [...] la formación debe servir a los objetivos de competitividad de las empresas y estar bajo su exclusiva dirección, mientras que para los sindicatos [...] se trata de asegurar el aprendizaje de todos los trabajadores, especialmente de los menos cualificados, que garantice su promoción y movilidad profesional más allá, incluso, de la propia empresa, al tiempo que reclaman su participación en la programación y gestión de la oferta formativa. Los Estados, por su parte, adoptan una función mediadora y, acogiéndose al principio de subsidiariedad, tratan de derivar hacia las partes la financiación de los programas, reservándose, según los casos, algunas competencias formales" (ibidem: 25-26). De hecho, la empresa que invierte en la cualificación de sus trabajadores sólo suele plantearse la cuestión a corto plazo y en relación con la necesidad de adquirir capacidades muy específicas y directamente utilizables en el puesto de trabajo, planteamiento que puede estar en contradicción no sólo con los intereses de los trabajadores sino también con las estrategias de modernización a largo plazo del sistema productivo en su conjunto. En todo caso, debe dejarse constancia de la poca atención que las empresas españolas han dedicado tradicionalmente a mejorar la cualificación de sus trabajadores: "El gasto medio de las empresas españolas [en FPC], según la Encuesta de Costes Laborales (INE), se sitúa en el 0’2\% del coste laboral total mientras que la media de la Unión Europea se sitúa en el 1'5\%" (J. Castillo et al., pp. 8). 
RIS

REVISTA INTERNACIONAL DE SOCIOLOGIA

NN30, Septiembre-Diciembre. 2001

ERIC SANCHIS

Finalmente, la determinación del tiempo necesario para alcanzar un nivel de cualificación dado también presenta complicaciones. Es automática en aquella parte del proceso de cualificación que transcurre en dispositivos formales, pero es problemática en aquella otra parte que coincide con el proceso mismo de trabajo: más fácil cuanto más estandarizados estén los procedimientos, más difícil cuanto más importante sea la dimensión artesanal de la actividad laboral.

\section{CAMBIO TÉCNICO, TERCIARIZACIÓN Y CUALIFICACIÓN}

En el debate secular originado en torno a la relación entre cualificación y progreso técnico han surgido dos grandes paradigmas: el optimista y el pesimista. El primero alcanza su formulación más rigurosa en la teoría del capital humano: el progreso tecnológico, y por tanto la creciente complejidad del aparato productivo, implica un mayor nivel de utilización de trabajo cualificado al crear empleos más cualificados que los que destruye y aumentar la cualificación de una parte significativa de los que se mantienen. La incorporación de las nuevas tecnologías de la información a los procesos productivos habría reforzado estas tendencias. El segundo es sobre todo de matriz marxista y tiene un punto de referencia obligado en el libro de $\mathrm{H}$. Braverman sobre Estados Unidos, cuyo expresivo subtítulo no deja lugar a dudas en cuanto a su contenido. La historia del progreso técnico bajo el capitalismo es la historia de una peculiar profundización en la división del trabajo que entraña la descualificación creciente de la mayoría de los trabajadores. El trabajador cualificado es más caro y tiene más poder de control sobre el proceso productivo. El objetivo del cambio técnico es arrebatarle la cualificación para transferirla a la máquina y así debilitar su posición de fuerza en el conflicto industrial. Frente al determinismo tecnológico recualificador de la tesis optimista, la tesis marxista coloca el determinismo descualificador de la lógica del modo de producción capitalista.

La investigación posterior a Braverman ha complejizado los términos del debate y ha puesto de manifiesto las dificultades que debe afrontar todo intento de descubrir tendencias generales de uno u otro signo. En realidad es probable que los procesos fatales y generalizados de promoción o degradación del trabajo sólo se encuentran en los libros, como han señalado A. Accornero y F. Carmignani (1986:171). Esas dificultades tienen su origen, al menos, en dos tipos de problemas. Primero, al comparar la estructura del empleo según las cualificaciones en dos estadios sucesivos de progreso técnico pueden ocurrir todas estas cosas: 1) algunos empleos desaparecen, 2) aparecen empleos nuevos, 3) algunos empleos se mantienen con características invariables, 4) algunos empleos permanecen pero sufriendo cambios significativos entre un estadio y otro. Puede analizarse el nivel de cualificación de los empleos que desaparecen en relación con los nuevos empleos y puede analizarse, si es el caso, el sentido 
del cambio en los empleos que permanecen. Cuando los diferentes elementos que integran la cualificación sean básicamente los mismos, el problema se reducirá a ponderar el nuevo peso relativo de cada uno de ellos. Pero si el cambio afecta sobre todo a la composición de la cualificación el análisis se complica enormemente. Segundo, el paso de un estadio de progreso técnico a otro va inextricablemente acompañado de un desplazamiento del empleo desde la industria a los servicios. Y este desplazamiento entraña, precisamente, sobre todo cambios en la composición de la cualificación. Lo que se pretende explicar es que, antes que empleos más o menos cualificados, lo que encontramos son empleos con cualificaciones diferentes: empleos donde la cualificación técnica se define de otra manera e incluye habilidades sociales cada vez menos prescindibles, donde la cualificación cultural cada vez puede darse menos por descontada, donde el proceso de adquisición de las cualificaciones tácitas va a verse modificado por los cambios que ha conocido el proceso de socialización de las nuevas generaciones.

Si además el paso de un estadio técnico a otro se produce al mismo tiempo que cambian las características biosociológicas de la fuerza de trabajo (sexo, edad, etnia, niveles educativos, culturas del trabajo, etc.) y mientras cambia la correlación de fuerzas entre capital y trabajo (todo esto ha ocurrido durante los últimos veinticinco años), el esfuerzo investigador deviene verdaderamente arduo. En cualquier caso el hecho cierto es que sabemos mucho menos acerca de los nuevos empleos terciarios que de los viejos empleos industriales.

El análisis de la evolución de las cualificaciones puede abordarse desde dos perspectivas metodológicas, que denominaremos macro y micro. En todo caso debe diferenciarse claramente entre el análisis del nivel y evolución de la cualificación de los trabajadores y el de los puestos de trabajo. Técnicamente, la aproximación macro a las cualificaciones de los trabajadores - por ejemplo mediante el estudio de datos censales - es la que menos complicaciones presenta. Suele utilizarse como indicador las titulaciones educativas o, simplemente, los años transcurridos en el sistema educativo. Este último se utiliza sobre todo a efectos de comparaciones internacionales, dadas las dificultades que plantea la equiparación de titulaciones emitidas por sistemas educativos estructurados de acuerdo con sus propias especificidades nacionales y con unas evoluciones históricas fuertemente diferenciadas. Desde este punto de vista, en las sociedades desarrolladas y en vías de desarrollo la evolución ha sido obviamente positiva. Ahora bien, de datos como el aumento de los titulados universitarios, la reducción de número de analfabetos, las dificultades crecientes de los trabajadores menos educados en el mercado de trabajo o las exigencias crecientes de los empleadores, en cuanto a credenciales educativas, a la hora de contratar trabajadores, es muy arriesgado deducir conclusiones sobre la evolución de la cualificación de los empleos. 
La aproximación macro a la cualificación de los empleos - por ejemplo a partir de las encuestas de población activa o de los mismos censos- es más problemática. El informe elaborado por L.J. Garrido et al., (1991), que pretendía analizar las necesidades de la estructura ocupacional española y su evolución, es una buena muestra de las dificultades a las que hay que enfrentarse. Tanto las encuestas como los censos - en cada país - se basan en las correspondientes clasificaciones nacionales de ocupaciones. Ahora bien, tales clasificaciones son, al mismo tiempo y de manera inevitable, un repertorio de empleos y de profesiones. Los empleos son definidos sobre todo por la empresa. Las profesiones son definidas sobre todo por los trabajadores (por sus organizaciones sindicales y profesionales), pero también por otras instancias que tienen en cuenta factores como la estructura del sistema educativo. Y el hecho es que tanto la estructura de los empleos como la de las profesiones reflejan no sólo diferencias de cualificación sino también, por ejemplo, diferencias salariales o de autoridad. En resumen, una ocupación es al mismo tiempo un empleo (que remite a cualificaciones nominales) y una profesión (que remite a cualificaciones efectivas). Por tanto, al analizar la evolución de la cualificación de los empleos a partir de las ocupaciones, se corre el peligro de acabar analizando (de nuevo) la evolución de la cualificación de los trabajadores.

En cualquier circunstancia, la aproximación macro a la cualificación de los empleos a partir de las ocupaciones permite cuando menos analizar la evolución de los efectivos de cada ocupación. Por ejemplo, si aumenta o disminuye el número de directivos de grandes empresas, altos funcionarios, mecánicos de precisión, camareros, enfermeros o peones de la industria. Pero, como señala M. Fernández Enguita (1995:85), tiene el inconveniente de que no capta "los cambios dentro de cada ocupación, suponiendo constantes en el tiempo sus características". Desde esta perspectiva, a veces se ha enfatizado el hecho de que mientras las ocupaciones de mayor nivel, en algunos países, son las que más crecen en términos relativos, las de menor nivel no crecen o incluso pierden peso. De donde se deduce una lenta tendencia al aumento de las cualificaciones. Sin embargo, esta conclusión debe ser contemplada con cautela. Primero, porque el incremento del empleo en las ocupaciones más cualificadas suele ser muy modesto en términos absolutos; y segundo porque en el amplísimo surtido de ocupaciones de nivel intermedio se producen movimientos de signo opuesto que arrojan dudas sobre el signo de la tendencia dominante. Además, no debe descartarse una tendencia a la polarización de las cualificaciones compatible con un mayor peso de las de alto nivel. Como han señalado Lope y Martín Artiles (1993), esta tendencia puede ser reforzada por la propia política de personal de las empresas, que suelen concentrar la inversión en formación en la minoría de trabajadores previamente más cualificados. En cualquier circunstancia, si hacemos abstracción de los problemas de homologación de las diferentes clasificaciones nacionales de ocupaciones, 
comparar el peso que ocupan los empleos de cualificación máxima y mínima en varios países en un momento dado, no deja de ser un ejercicio útil.

Otra técnica de aproximación macro es la de preguntar mediante encuesta a una muestra representativa de la población acerca de las características de los empleos que ocupan. Así se hizo en España, en 1985, con la llamada Encuesta sobre condiciones de vida y trabajo, realizada por encargo de la Administración (VV. AA., 1988:86-87). El 49'3\% de los asalariados declaró que para hacer su trabajo no necesitaba ninguna cualificación formal (estudios); y el $20{ }^{\prime} 1 \%$, que no necesitaba ningún período de adaptación en el tajo (porcentaje que sube hasta el 51'2 si se considera a todos los que declararon necesitar menos de tres meses). Estas y otras preguntas sobre el tema, repetidas periódicamente, podrían dar una buena idea de la evolución de las cualificaciones. En sentido similar, la Encuesta de Calidad de Vida en el trabajo 1999 (Ministerio de Trabajo y Asuntos Sociales) incluye una pregunta al objeto de clasificar a los trabajadores asalariados según la relación existente entre el empleo ocupado en la actualidad y el nivel de estudios y experiencia. Casi el $95 \%$ responde que su nivel de estudios es suficiente o más que suficiente; el $90 \%$ responde que su experiencia es suficiente o más que suficiente. Dado el bajo nivel educativo medio de la población española, estas respuestas permiten hacerse una idea acerca del bajo nivel de cualificación de la mayoría de los empleos del mercado de trabajo español. En todo caso, esta técnica de aproximación plantea el problema metodológico de hasta qué punto la opinión de quien ocupa un empleo refleja la cualificación "objetiva" de éste.

Pero la perspectiva analítica en principio metodológicamente más adecuada es la de tipo micro, que consiste en el estudio en profundidad de un grupo restringido de empleos. Las investigaciones de este tipo tienen el inconveniente de que sus conclusiones no pueden generalizarse automáticamente. Una de las más conocidas - que combina técnicas cualitativas y cuantitativas-es la llevada a cabo por H. Kern y M. Schumann (1989). Pero la única conclusión general que se puede obtener tanto de este trabajo como de otros muchos estudios de casos es que la evolución de las cualificaciones laborales no está tecnológicamente determinada. El resultado final depende también de otras variables, como la cultura empresarial, que va a condicionar fuertemente que las unidades productivas (ante los desafios de la competitividad) se dejen arrastrar por la inercia de las soluciones fordistas o evolucionen hacia fórmulas más imaginativas, o la capacidad de los trabajadores organizados para negarse a aceptar determinadas condiciones de trabajo. De hecho, como apunta Wood, una de las críticas más compartidas a la tesis de Braverman es que, al limitarse en su análisis a la llamada dimensión objetiva del desarrollo capitalista, desestimó el impacto de la resistencia obrera. Quizás sea la evolución histórica que ha venido experimentando el trabajo de estiba en todos los puertos del mundo desarrollado, el ejemplo más claro de cómo la acción sindical puede acabar imponiendo una 
RIS

RETISTA INTERNACIONAL DE SOCIOLOGIA

№30. Septiembre-Diciembre, 2001

definición social de su cualificación que, a su vez, determina una modificación al alza de su cualificación técnica.

Así pues, la investigación empírica reciente obliga a matizar la macrovisión pesimista: aunque durante gran parte del siglo XX ha habido procesos descualificadores muy fuertes, la crisis del fordismo ha puesto de manifiesto que las ganancias de productividad no pueden seguir apoyándose en equipos cada vez más sofisticados y trabajadores pasivos y semiidiotizados. Pero sobre todo ha puesto en cuestión la macrovisión optimista. La transformación tecnológica de una empresa bostoniana de fabricación de pan, estudiada por R. Sennett (2000:66-78), es una ulterior confirmación de las insuficiencias de este enfoque. Vale la pena resumir brevemente las conclusiones de este trabajo, porque ilustra muy bien la diversidad de variables intervinientes.

En su primera visita a la fábrica, Sennett se encontró con un grupo de trabajadores, en su mayoría hombres adultos de origen griego, sindicados, hijos de panaderos que habían trabajado para la misma empresa y que dejaron el empleo para jubilarse (los puestos de trabajo habían pasado de padres a hijos a través del sindicato local). El trabajo se desarrollaba en un ambiente duro: las manos de los panaderos se sumergían constantemente en la harina y el agua, a menudo se quemaban con el horno, la amasadora primitiva requería mucha fuerza, el olor a levadura se mezclaba con el del sudor humano en las salas calientes, el calor hacía vomitar con frecuencia a los trabajadores, el trabajo era nocturno. Además del esfuerzo físico era imprescindible el trabajo en equipo, una solidaridad que alimentaba la conciencia de grupo reforzada por la identidad étnica: la preparación del pan era un ejercicio coreográfico que requería años de entrenamiento para salir bien; los hombres usaban la nariz y los ojos para decidir cuándo estaba listo el pan. Aquellos trabajadores tenían un fuerte orgullo del oficio, aunque no disfrutaban con su trabajo.

Veinticinco años después, la informatización del proceso de trabajo ha eliminado el contacto físico con los ingredientes y el producto. Ahora se utilizan máquinas complejas y reconfigurables para hacer un producto ultradiversificado de acuerdo con los principios de la especialización flexible. Los trabajadores supervisan el proceso mediante pantallas de ordenador organizadas en ventanas. Cualquier producto es posible "con sólo acercar un dedo a la pantalla. El pan se ha convertido en una representación en pantalla". La mayoría de los trabajadores ni siquiera ven el pan que fabrican y "en realidad los panaderos ya no saben cómo se hace el pan. [...] Los trabajadores dependen de un programa informático $\mathrm{y}$, en consecuencia, no pueden tener un conocimiento práctico del oficio". En cambio, ahora la panadería es asombrosamente fresca; bajo las relajantes lámparas fluorescentes, todo tiene un aspecto extrañamente silencioso. El ambiente de trabajo se parece más al de una oficina que al de una fábrica, y la calidad del producto es excelente.

Pero no es la tecnología lo único que ha cambiado. Ahora hay muchas 
mujeres, jóvenes y una gran diversidad étnica. El poder sindical se ha reducido en perjuicio de los trabajadores más jóvenes. El antiguo turno de noche ha sido reemplazado por una jornada mucho más flexible; los trabajadores van y vienen a lo largo del día; la panadería es una compleja red de horarios a tiempo parcial para las mujeres e incluso para algunos hombres. La conciencia de grupo y la identidad laboral son débiles: "En casa sí que hago pan, soy panadero. Aquí aprieto botones", afirma un trabajador; "hacer pan, zapatos, trabajos de imprenta, pídame lo que quiera", le dice una trabajadora a Sennett riendo. La mayoría de los trabajadores se van antes de cumplir los dos años ("no voy a hacer esto el resto de mi vida"), y los jóvenes no sindicados son los que duran menos. Para ser contratados, los trabajadores han de demostrar que saben manejar ordenadores, pero luego lo único que hacen es apretar botones en un programa de Windows diseñado por otros. "Hay una base económica - concluye Sennett-para estas mezcladoras, amasadoras y hornos fáciles de usar: permiten a la empresa contratar trabajadores con salarios inferiores que en el pasado, cuando los trabajadores, no las máquinas, eran los cualificados, aunque ahora todos tienen cualificaciones técnicas altas y certificadas".

No se pretende acabar mitificando románticamente todo pasado mejor ni dejarse caer ingenuamente en los brazos de la macrovisión pesimista. Al margen del caso estudiado, el mismo Sennett reconoce que "a niveles más altos del trabajo técnico, el advenimiento del ordenador ha enriquecido el contenido de muchos trabajos". Pero sí se pretende distinguir claramente entre varias cuestiones que suelen mezclarse dentro de la macrovisión optimista. Una cosa es reconocer que el mantenimiento de la competitividad de las economías avanzadas y el diseño de medios de producción y productos cada vez más complejos depende de manera crítica del trabajo de científicos de excelencia en universidades, institutos de investigación y departamentos de $\mathrm{I}+\mathrm{D}$ de las empresas, dotados con unos recursos que en el caso español habrían de duplicarse (desde menos del $1 \%$ hasta el $2 \%$ del PIB). Otra cosa muy distinta es deducir de todo ello que la mayoría de quienes van a aplicar esos progresos en los empleos correspondientes necesitan cualificaciones más elevadas para hacerlo. Sería como decir que afeitarse con una maquinilla eléctrica requiere habilidades superiores a hacerlo con una navaja, cuando en realidad ocurre todo lo contrario. Porque una cosa es producir progreso científico y tecnológico y otra aplicarlo en el trabajo.

El desplazamiento sectorial del empleo ha contribuido a alimentar el mito de la recualificación. Tradicionalmente se ha considerado que el crecimiento del empleo terciario implica de manera más o menos automática una reducción del trabajo fisicamente duro y un aumento de las cualificaciones. En el éxito de esta interpretación han confluido el hecho cierto de la automatización de muchas tareas penosas y repetitivas, las formulaciones académicas sobre la sociedad postindustrial (en la que el conocimiento es el primer factor productivo), las representaciones populares (en parte fundamentadas) sobre la calidad del trabajo 
RIS

REVISTA INTERNACIONAL DE SOCIOLOGÍA

№ 30, Septiembre-Diciembre, 2001

en los servicios y el mayor desconocimiento de la realidad del empleo terciario propiciado por la mayor atención que la sociología ha prestado tradicionalmente al trabajo industrial.

Aquí es necesario de nuevo distinguir entre fenómenos que tienden a confundirse. En los servicios se está consolidando un amplio espectro de actividades laborales fuertemente diversificadas en cuanto a cualificaciones y en cuanto a condiciones de trabajo. Son muchos los ejemplos merecedores de una reflexión que aquí sólo se esboza. Así, muy probablemente la creciente sofisticación tecnológica del medio urbano ha recualificado el trabajo en los servicios contra incendios, aunque en este caso la relación entre progreso técnico y cualificación no puede entenderse a la manera tradicional. Puede intuirse igualmente que el trabajo de policía se ha recualificado, pero no a causa del mayor contenido tecnológico de los medios de represión sino porque en una democracia avanzada el objetivo de las fuerzas de orden público no puede ser eliminar al infractor sino sólo neutralizarlo. En el otro extremo del espectro, la aproximación periodística que ha realizado L. Fernández Zaurín (1998) a los empleos basura del nuevo terciario español pone al descubierto todo un mundo de trabajo precario (actividades relacionadas con el ocio nocturno, mensajeros, repartidores de pizzas a domicilio, establecimientos de comida rápida, vigilantes de seguridad) que cuestiona de manera radical el paradigma de la sociedad postindustrial. En efecto, muchas de estas actividades no requieren especiales cualificaciones y el esfuerzo fisico sigue presente o bien ha sido sustituido por la tensión nerviosa o el hastío (en 1988 se suicidaron treinta y ocho vigilantes de seguridad). El paro masivo y el escaso poder sindical han alumbrado unas condiciones de trabajo que muchas veces son ya historia en el empleo parcelado de la industria. La bajísima identificación con el trabajo (nadie quiere jubilarse de eso), la juventud de casi todos los trabajadores y la elevada rotación en el empleo dibujan un panorama que no va a ser fácil modificar.

No obstante, en relación con algunos de estos nuevos empleos postindustriales, a poco que se reflexione surge la duda de si sus "competencias objetivamente definidas" son realmente tan bajas como se presume o lo que es bajo es el reconocimiento social de las cualificaciones necesarias para desempeñarlos, derivado de la débil posición de quienes los ocupan en el mercado de trabajo, y por tanto en el proceso de construcción social de la cualificación. Compárense, por ejemplo, las condiciones de trabajo, contractuales y salariales del joven motorista repartidor de pizzas a domicilio con las del estibador adulto y sindicado, empleo este último que ha ido ganando consideración a lo largo de la historia del movimiento obrero. ¿Es realmente menor la cualificación del primer empleo?

El caso del empleo de teleoperador en los call centres (Taylor y Bain, 1999; Bain y Taylor, 2000) es un ejemplo paradigmático de cómo puestos de trabajo ocupados por trabajadores dotados de cualificaciones efectivas al 
menos medio-altas (incluyendo muchas veces la licenciatura universitaria) son desempeñados en un marco de condiciones de trabajo, salariales y contractuales muy duras y definidos nominalmente de cualificación más bien baja. Estos centros de trabajo que algunos han caracterizado como una especie de nuevos sweatshops donde la cadena de montaje está grabada en la mente del trabajador, han experimentado un fuerte crecimiento en el Reino Unido durante los últimos años, empiezan a ser conocidos en España y en la actualidad ocupan ya probablemente a más de 200.000 trabajadores en toda la Unión Europea. Convertidos durante cuatro, seis u ocho horas diarias en "meros sucedáneos de una máquina parlante", los teleoperadores se ven obligados a soportar una actividad embrutecedora y rutinaria que no reclama sino rechaza cualquier implicación intelectual: "Durante un día normal de trabajo cada teleoperador atendía entre veinte y veinticinco llamadas por hora, con lo que un ciclo completo de trabajo oscilaba entre los dos minutos y los dos minutos y medio" (A. del Bono, 2000:10). Es absolutamente estéril intentar explicar esta situación en clave de nuevas tecnologías, pues sólo puede ser entendida en función de opciones empresariales sobre nuevas formas de organización del trabajo que sólo han podido ser adoptadas en el nuevo marco de relaciones laborales que se va imponiendo en las sociedades avanzadas desde hace ya tres décadas y ante las que sólo recientemente y con todas las dificultades empiezan a oponerse nuevas formas de resistencia.

El caso de los call centres ilustra también la complejidad del concepto de cualificación y permite vaticinar que seguirá discutiéndose durante los próximos años. Previsiblemente en esta discusión ganará importancia la distinción entre competencia profesional y cualificación, entendiendo la primera como lo que un individuo concreto es capaz de hacer efectivamente en un puesto de trabajo, y la segunda como certificación oficial y reconocimiento formal de la primera (J. Planas et al., 2000). Y se hará más evidente el papel que desempeñan las cualificaciones tácitas en la adquisición de la competencia, así como la necesidad de analizar las relaciones entre cualificación, flexibilidad funcional y polivalencia.

Como el sistema educativo continuará desarrollándose, el debate sobre las relaciones entre educación y cualificación ganará en intensidad, y tras éste seguirá habiendo dos orientaciones parcialmente contrapuestas de la educación que habrá que intentar conciliar: una centrada en los derechos del ciudadano-trabajador y otra centrada en las necesidades de la empresa. Intento que seguirá plagado de dificultades porque seguirá existiendo una contradicción entre la aspiración ciudadana a más educación, más bienestar y más igualdad de oportunidades, y la realidad de un mercado de trabajo en el que seguirá habiendo paro, empleos degradados e inmigrantes (ciudadanos de segunda clase) dispuestos a ocuparlos. 
R I S

RETISTA INTERNACIONAL DE SOCIOLOGLA

N` 30. Septiembre-Diciembre. 2001

ERIC SANCHIS

\section{CONCLUSIÓN}

El empleo es una relación social, y las diversas capacidades necesarias para desempeñarlo con eficacia no pueden ser conceptualizadas en clave puramente técnica, pues incluyen habilidades relacionales y culturales. En las sociedades tradicionales, de capitalismo temprano o de pleno empleo, el acceso al trabajo era más bien automático y el concepto de cualificación podía reducirse a su dimensión estrictamente técnica en la medida en que las capacidades no técnicas podían darse por descontadas.

En las sociedades postindustriales, el paro estructural, el alejamiento de la población trabajadora del nivel de subsistencia, la quiebra de la relación directa entre familia y empleo, la creciente distancia social entre estas agencias tradicionales de cualificación, el desarrollo del sistema educativo y otros dispositivos formativos como nuevas agencias cualificadoras, las dificultades de estas nuevas agencias para enganchar al empleo a los parados de larga duración y la incapacidad del mercado de trabajo para responder a las expectativas de movilidad social de las nuevas generaciones, han problematizado el acceso al empleo y han puesto en evidencia las limitaciones de una definición reduccionista de la cualificación. Junto con el acceso al empleo se problematizan también el proceso de adquisición de la cualificación y la conceptualización de ésta. Ahora las capacidades no técnicas ya no pueden darse por descontadas.

La necesidad de considerar explícitamente este tipo de capacidades a la hora de diseñar los diferentes dispositivos de erogación de cualificaciones, parece particularmente clara en relación con el empleo en los servicios y con las estrategias de las unidades productivas para mantener la competitividad y poder hacer frente a los retos de la globalización; pues la competitividad depende cada vez más de la disposición de los trabajadores a colaborar lealmente en la consecución de los objetivos de la empresa. Pero en la medida en que la cualificación no está determinada sólo tecnológicamente sino que es asimismo una construcción social resultado de las estrategias emprendidas por los diferentes grupos que intervienen en el proceso productivo para defender sus posiciones de poder, esa colaboración requiere también la profundización de la democracia industrial.

\section{REFERENCIAS BIBLIOGRÁFICAS}

ACCORNERO, A. y F. CARMIGNANI (1986), I paradossi della disoccupazione, Bolonia, Il Mulino.

ARBIZU, F. (2000), “CCómo se adquieren las cualificaciones?”, en G. TORRES y F. J. HERNÀNDEZ (eds.), Los sistemas de cualificación profesional, Alzira (Valencia), Germania, pp. 29-34. 
ARIĖS, Ph. (1973), L'enfant et la vie familiale sous l'Ancien Régime, París, Seuil.

BAIN, P. y P. TAYLOR (2000), "Entrapped by the 'electronic panopticon'? Worker resistance in the call centre", New Technology, Work and Employment, vol. 15, n. ${ }^{\circ}$ 1, pp. 2-18.

BENEYTO, P. J. y P. GUILLÉN (1998), "La Formación Profesional en España: expansión y cambio", en P. J. BENEYTO y P. GUILLÉN (eds.), Formación Profesional y empleo: la construcción de un nuevo modelo, Alzira (Valencia), Germania, pp. 11-39.

BRAVERMAN, H. (1974), Labor and Monopoly Capital. The Degradation of Work in the Twentieh Century, Nueva York, Monthly Review Press. [Se sugiere leer la introducción de J. B. Foster a la nueva edición de 1998]

CASTILLO, J.J. et al. (2000), "División del trabajo, cualificación, competencias. Una guía para el análisis de las necesidades de formación por los trabajadores", Sociología del Trabajo, n. ${ }^{\circ} 40$, pp. 3-50.

CASTILLO MENDOZA, C.A. y E. TERRÉN LALANA (1994), "De la cualificación a la competencia: elementos para una reconstrucción epistemológica", Cuadernos de Relaciones Laborales, n. ${ }^{\circ}$, pp. $75-91$.

COLLER, X. (1997), La empresa flexible. Estudio sociológico del impacto de la flexibilidad en el proceso de trabajo, Madrid, CIS-Siglo XXI.

COLLINS, R. (1989), La sociedad credencialista. Sociología histórica de la educación y la estratificación, Madrid, Akal.

CRUBELLIER, M. (1979), L'enfance et la jeunesse dans la société française, 1800-1950, París, Armand Colin.

DEL BONO, A. (2000), "Call centers, ¿el trabajo del futuro? El caso de Estrategias Telefónicas S. A. (ESTRATEL)", Sociologia del Trabajo, n. ${ }^{\circ} 39$, pp. 3-31.

FERNÁNDEZ ENGUITA, M. (1995), La escuela a examen, Madrid, Pirámide.

FERNÁNDEZ ZAURÍN, L. (1998), Y tú de qué trabajas, Barcelona, DVD Ediciones.

FRIGOLÉ, J. (1998), Un hombre. Género, clase y cultura en el relato de un trabajador, Barcelona, Muchnik Editores.

GARCIA CALAVIA, M.A. (1999), "La herencia de Harry Braverman", Revista Internacional de Sociologia, n. ${ }^{\circ} 24$, pp. 139-167.

GARCÍA ESPEJO, I. (1999), "Formación en el trabajo y movilidad laboral", Papers, n. ${ }^{\circ} 59$, pp. 195-219.

GARRIDO, L. J. et al. (1991), Prospectiva de las ocupaciones y la formación en la España de los noventa, Madrid, Instituto de Estudios y Análisis Económicos. 


\section{RIS}

GÓMEZ BUENO, C. (2000), "Del etiquetado de las ocupaciones según nivel de cualificación", Sociología del Trabajo, n. ${ }^{\circ}$ 39, pp. 33-61.

HANDMAN, M.H. (1983), La violence et la ruse. Hommes et femmes dans un village grec, Aix-en-Provence, Édisud.

INSTITUTO DE LA JUVENTUD, Informe Juventud en España 2000, Madrid, edición electrónica, Ministerio de Trabajo y Asuntos Sociales.

KERN, H. y M. SCHUMANN (1989), El fin de la división del trabajo. Racionalización en la producción industrial, Madrid, Ministerio de Trabajo.

LOPE, A. y A. MARTÍN ARTILES (1993), "Cambio técnico y recualificación. Formación y adquisición de las cualificaciones en la empresa. Un estudio de casos", Sociología del Trabajo, n. ${ }^{\circ}$ 19, pp. 67-97.

MARUANI, M. (1991), "La construcción social de las diferencias de género en el mercado de trabajo", Revista de Economia y Sociología del Trabajo, n. ${ }^{\circ}$ 13-14.

MIETTO, M. y M. G. RUGGERINI (1988), Storie di fabbrica. Operai metallurgici a Reggio Emilia negli anni '50, Turin, Rosenberg \& Sellier.

MINISTERIO DE TRABAJO Y ASUNTOS SOCIALES, "Encuesta de Calidad de Vida en el Trabajo 1999", Madrid, edición electrónica.

MONCADA, A. (1979), La adolescencia forzosa, Barcelona, Dopesa.

MUÑOZ MOLINA, A. (1991), El jinete polaco, Barcelona, Planeta.

OFFE, C. (1977), "Sistema educativo, sistema occupazionale e politica dell'educazione. Per una definizione della funzione sociale complessiva del sistema educativo", en Lo stato nel capitalismo maturo, Milán, Etas Libri, pp. 159-203.

PLANAS, J. et al. (2000), Marché de la compétence et dinamiques d'ajustement, Toulouse, LIRHE.

SANCHIS, E. (1991), De la escuela al paro, Madrid, Siglo XXI.

SENNETT, R. (2000), La corrosión del carácter. Las consecuencias personales del trabajo en el nuevo capitalismo, Barcelona, Anagrama.

STREECK, W. (1992), "Training and the new Industrial Relations: a Strategic Role for the Unions", en M. REGINI (ed.), The Future of Labour Movements, Londres, Sage.

TAYLOR, P. y P. BAIN (1999), “'An Assembly Line in the Head': Work and Employee Relations in the Call Centre", Industrial Relations Journal, vol. 30, n. ${ }^{\circ}$, pp. 101-117.

THOMSON, E. P. (1984), Tradición, revuelta y consciencia de clase, Barcelona, Crítica. 
TOURAINE, A. (1963), "La organización profesional de la empresa", en G. FRIEDMANN, y P. NAVILLE, Tratado de sociología del trabajo, México, Fondo de Cultura Económica, vol. 1, pp. 384-427.

VERNIĖRES, M. (1978), "Essai sur le rôle de la qualification des travailleurs dans le fonctionnement du marché du travail", en VV. AA., Les marchés locaux du travail, Paris, La documentation française, pp. 19-37.

VV. AA. (1988), Análisis de las condiciones de vida y trabajo en España, Madrid, Ministerio de Economía.

WOOD, S. (1994), "El debate sobre la descualificación. Las nuevas tecnologías y la organización del trabajo", en L. FINKEL, La organización social del trabajo, Madrid, Pirámide, pp. 295-307. 